CIC. Cuadernos de Información y Comunicación

ISSN: $1135-7991$

\title{
El anteproyecto de ley general de comunicación audiovisual: una guía crítica de lectura
}

\author{
A Enrique Bustamante, \\ maestro y amigo.
}

\author{
Ramón Zallo Elguezábal ${ }^{1}$ \\ Enviado: 14-05-2021 / Evaluado: 24-05-2021 / Aceptado: 26-05-2021
}

Resumen. El periodo de alegaciones institucional y de agentes al Anteproyecto de Ley General de Comunicación Audiovisual ha sido muy rico en aportaciones. En esta guía crítica se hace un seguimiento temático de cambios y continuidades respecto a la Ley vigente desde una clave interpretativa de avances y retrocesos en relación al servicio público, los derechos ciudadanos y de los usuarios, la diversidad, el pluralismo, menores, género, publicidad, obra audiovisual, la gobernanza y otros parámetros.. para llegar a un diagnóstico final que es muy crítico con el anteproyecto aun reconociendo exhaustivamente las novedades que recoge.

Palabras clave: ley general de comunicación audiovisual; anteproyecto; pluralismo; diversidad

\section{[en] The Draft of General law of audiovisual comunication: a critical reader}

\begin{abstract}
The period of institutional and agent allegations to the draft of the General Law of Audiovisual Communication has been very rich in contributions. In this critical guide; a thematic monitoring of changes and continuities with respect to the current Law is made from an interpretative key of the advances and setbacks in relation to public service; citizen and user rights; diversity; pluralism; minors; gender; advertising; audiovisual work; governance and other parameters... to arrive at a final diagnosis that is very critical of the preliminary draft; even though it exhaustively recognizes the novelties it contains.
\end{abstract}

Keywords: General Law of Audiovisual Communication; draft; pluralism; diversity.

Sumario. 1. Aspectos de interés del APL. 2. Aspectos criticables del APL. 3. Diagnóstico general. Referencias

Cómo citar: Zallo Elguezábal, R.(2021), El anteproyecto de ley general de comunicación audiovisual: una guía crítica de lectura, en CIC. Cuadernos de Información y Comunicación 26, 149-160.

El Anteproyecto de Ley General de Comunicación Audiovisual (APL en adelante) se deriva de la obligación -ya fuera de plazo- de trasposición de la nueva Directiva europea de servicios de comunicación audiovisual (UE) 2018/180, si bien en el anteproyecto español también se regula la radio. Abarca por vez primera a las plataformas de intercambio de vídeo y detalla la normativa aplicable a los servicios

1 Departamento de Comunicación Audiovisual y Publicidad Facultad de Ciencias Sociales y de la Comunicación Universidad del País Vasco (UPV-EHU)

rammzallo@gmail.com 
a «petición» en la pretensión de sustituir a la vigente Ley 7/2010. Tiene siete títulos y 164 artículos.

Desde el punto de vista normativo, se trata de una mera y simple trasposición más que de un desarrollo legal para regular el complejo panorama comunicativo. Desde el punto de vista ideológico, es conservador y mercantilista y no lo es aún más, precisamente, por las restricciones europeas. Es sabido que el poder mediático en España es menos autónomo respecto a poderes económicos y políticos que en otros países con más tradición democrática. Desde el punto de vista material, resulta un modelo híbrido y neoliberal entre la Ley General de Comunicación Audiovisual de 2010 del Gobierno de Rodríguez Zapatero y que pilotó la Vicepresidenta María Teresa Fernández de la Vega (con la asesoría permanente de UTECA, el lobby de la TV privada) y que sigue vigente, más los añadidos - ratificados ahora- del Gobierno Rajoy (Comisión Nacional de Mercado y la Competencia, CNMC en adelante) como comisión reguladora competente en vez de un específico Consejo Estatal de Medios Audiovisuales (CEMA), la elección del Director General de RTVE pasó a ser por mayoría y no por $2 / 3$ y se autoriza la gestión privada de los Servicios Públicos autonómicos calificándola de "gestión indirecta".

Ya con la Ley de 2010 del Gobierno socialista el acento pasó "del servicio público a la concentración; de la descentralización a la centralización; de la extensión del conocimiento a su acumulación y apropiación desde el capital cognitivo; del modelo keynesiano -combinado con el schumpeteriano y una apuesta por el acceso social-a un regreso tardío al modelo neoliberal, tras haber distorsionado el mercado mediante decisiones públicas en beneficio de los prestadores de servicios audiovisuales más importantes" (Zallo 2011:301-321).

Ya era una normativa que potenciaba el capital cognitivo privado y su acumulación mediante concentración, a costa del pluralismo y la competencia, apropiándose en especial de los valores añadidos del capital humano y del capital social, y dentro de ellos del capital intelectual y cultural, además de rentas directas o indirectas del creciente consumo audiovisual colectivo.

Como ese paso ya se dio, el nuevo giro va en la misma dirección neoliberal (Bustamante 2020) con condiciones, incluye en la ecuación una nueva y tímida regulación para los operadores globales que han puesto patas arriba el espacio y el mercado cultural y comunicativo en esta década transcurrida. En el APL se les teme y homologa.

\section{Aspectos de interés del APL}

Es más ordenado que la ley vigente y con más precisas definiciones de los sujetos y servicios (art. 2). Intenta responder a los operadores globales, a los nuevos contenidos audiovisuales con las mismas reglas de juego para cualquier tecnología utilizada por lo que propone una regulación (aunque mínima y tímida) de la selva, en torno a obligaciones de las plataformas de TV no lineales (a petición), de intercambio de vídeos y (se supone) de agregadores de servicios de comunicación audiovisual de terceros (Títulos IV y V). Se amplía el Registro a todo tipo de prestadores de servicios audiovisuales incluyendo, como novedad, a los antedichos, aunque solo tengan la obligación de inscripción que, en su caso, implicaría una autorización automática, siendo compatible con los Registros autonómicos. 
Mantiene obligaciones de programación de la ley vigente (reservar el 51\% del tiempo de emisión de TV convencional a obras audiovisuales europeas), y las extiende de forma restringida a las ofertas de las plataformas desde dentro o desde otros Estados (HBO, Netflix..), en los arts. 108 a 118. Incluye como una de las opciones para la financiación obligatoria de obra europea audiovisual la contribución directa al Fondo para la Protección de la Cinematografía (arts. 115 y 117).

En lo que respecta a derechos ciudadanos de sectores sociales, define los derechos de la mujer en comunicación (art. 6), con algunas aplicaciones en el resto de la normativa (información sexista, publicidad, eventos deportivos, informe anual sobre su representación en informativos). Una muestra de ello es que se incluyen algunos eventos deportivos femeninos como acontecimientos de interés general para su emisión en abierto (art. 144).

Asimismo, mejora la regulación y las obligaciones para la accesibilidad de las personas discapacitadas: fija porcentajes e indicadores de cumplimiento del subtitulado, audio-descripción y lengua de signos, vinculándolos a franjas horarias y géneros en los que insertar las medidas de accesibilidad (arts. 83, 99 a 107, 149, disposiciones adicional y final).

Los servicios públicos (estatal o autonómico), con pocas novedades regulatorias, estarán, sin embargo, sujetos a test de valor público cuando, en el correspondiente espacio comunicativo, se pretendan nuevos servicios o canales que no estén contemplados en sus mandato-marcos y contrato-programas vigentes. Asimismo, para alguna actividad, deben poner en marcha indicadores de rentabilidad social (art. 149).

Conceptualmente, por exigencia europea, trae como novedad el concepto de prominencia en la visibilidad de obligaciones, aunque solo se le mencione para el caso de audiodescripciones para personas discapacitadas en programas televisivos a petición y para obras europeas en catálogos de a petición (art. 114).

Igualmente, se mandaría extender la autorregulación y corregulación (arts. 12, 13 y sus aplicaciones en otros artículos) en distintos campos; y sería preceptiva como obligación gubernamental, a partir de la ley, un Plan estratégico audiovisual en el Estado Español cada 6 años (art. 148).

Según la exposición de motivos el desarrollo legislativo territorial es competencia autonómica, aunque con perfiles difusos. También se señala que las Comunidades Autónomas pueden elevar las exigencias del APL sobre sus servicios públicos en lo relativo a financiación de obra audiovisual (art. 116.3).

Aunque no se amplían las competencias autonómicas, y en algún caso se invaden, se invita a que las Comunidades Autónomas sean quienes regulen la televisión y radio sin ánimo de lucro para hacer efectivos los arts. 48 y 80 . Sin embargo, siguen dependiendo de una planificación previa y de la disponibilidad de espectro, o sea, de la voluntad política tanto central como autonómica, que no es mucha ya que las ven más como un problema que como una oportunidad social.

Se confirma el amparo de convenios entre Comunidades Autónomas limítrofes y con afinidades lingüísticas y culturales (art. 72). Se entiende que las autoridades autonómicas de comunidades con lengua propia puedan exigir a RTVE "Dar a conocer la diversidad cultural y lingüística de España" (art. 50. e). Del mismo modo, con el art. 42, y por ineludible prescripción de la UE, las comunidades vasca y catalana ven amparada una prestación transfronteriza abierta de servicios audiovisuales -con libertad de recepción, sin interferencias, con obligada planificación estatal posibili- 
tadora-para fortalecer las relaciones cultural-comunicativas a uno y otro lado de sus fronteras pirenaicas.

Mantiene en 15 años el período de duración de las licencias para prestar el servicio mediante ondas hertzianas terrestres, pero matiza el sistema de renovación automática e indefinida vigente (art. 28). Igualmente mantiene el horario de los programas esotéricos y juegos de azar y apuestas -con significativas excepciones en el caso de las entidades habilitadas- entre la 1:00 a 5:00 (arts. 82, 97 y 121).

\section{Aspectos criticables del APL}

Los aspectos criticables no lo son en el mismo grado.

\section{a) Continuidades sin avances ni retrocesos}

Ya en la anterior ley las RTV privadas dejaron de ser servicio público de gestión privada -con menores obligaciones, eso sí que las de gestión pública- para pasar a ser "servicios de interés general" siguiendo la doctrina europea dominante lo que, entre otras facultades, les permiten vender y arrendar licencias (no subarrendar) en ciertas condiciones (art. 31).

En lo relativo a concentración para adquirir otras empresas o grupos se mantienen los límites de concentración en televisión en el 27\% de la audiencia y se mantiene la posibilidad de superar ese porcentaje en el tiempo. Siguen estando los topes en 8 canales (2 multiplex) en el ámbito estatal y 4 (1 multiplex) en el autonómico. Aunque se continúa señalando que ha de haber un mínimo de tres prestadores, no pasa nada si son dos quienes dominan el mercado siempre que haya otro de menor peso en el mismo.

En el caso de las radios en cadena (art. 77) el anteproyecto no propone cambios (no más de 5 licencias en una misma demarcación, máximo de 1/3 en todo en Estado, $40 \%$ de licencias en cada Comunidad Autónoma). No incluye obligaciones de contenido locales ni mínimos de tiempo de desconexión. No entran en el cómputo las licencias asociadas, los acuerdos... con lo que la concentración de hecho en un triopolio omnipotente es mucho mayor de lo que da a entender la apariencia de las reglas. El anteproyecto pera desde una lógica de derechos adquiridos.

Para la televisión lineal se mantienen las cuotas (art. 113) del 51\% del tiempo de emisión anual neto ( ción para obra europea, reservándose su mitad al menos a obras en la lengua oficial del Estado o en alguna de las cooficiales. Un 10\% del tiempo de emisión anual se reservará para obra de producción independiente, cuya mitad al menos habrá sido producida en los últimos 5 años.

En el ámbito local de televisión se mantiene la barrera del 25\% de tiempo semanal de programación en cadena.

En cambio, los prestadores a petición tipo Netflix, HBO, Rakuten, Prime Video... han de reservar para obras europeas el 30\% de sus catálogos -con alguna obligación de prominencia, o sea, visibilidad-, y su mitad debe estar disponible en español o en lenguas cooficiales sin que tengan tampoco obligación expresa para hacerlo en estas últimas. Sin embargo, no aparecen como contribuyentes a la financiación de RTVE a diferencia de los canales abiertos o de pago (Movistar), lo que no parece razonable. 
Quizás se deje para una modificación del artículo 6 de la Ley 8/2009, de financiación de la Corporación de Radio y Televisión Española, que versa sobre la aportación de prestadores del servicio de televisión (ver postdata).

En la financiación de contenidos audiovisuales, los servicios públicos de ámbito estatal y autonómico (art. 116) deben destinar el 6\% de sus ingresos totales a financiar obra audiovisual europea, con un mínimo del $70 \%$ para obras de productoras independientes en la lengua del Estado y cooficiales, un mínimo del $45 \%$ para películas de productoras independientes y otro mínimo del 12\% para animación y documentales. Las CCAA están autorizadas a ampliar estas exigencias sobre sus televisiones públicas.

En el caso de la televisión privada lineal o a petición de ámbito estatal o autonómico, si tiene más de 10 millones de ingresos -quedan exentos los de ingresos menores a 10 millones- financiará anticipadamente obra audiovisual europea (art. 115). Si cuentan con más de 50 millones de ingresos anuales (art. 117) será el 5\% de los ingresos del año anterior los dedicados a financiar obra audiovisual europea, en las proporciones mínimas de $70 \%$ para productoras independientes y $40 \%$ para películas producidas en lengua oficial o cooficiales.

Ello merece algún comentario. Por una parte, no parece razonable que los prestadores con ingresos entre 10 y 50 millones -a pesar de que se les exima del $40 \%$ para películas- tengan el mismo porcentaje que los de más de 50 millones de ingresos, lo que haría conveniente una graduación descendente. Por otra, la contribución de los servicios a petición -sin carga de informativos ni programación generalista- a las obras audiovisuales, dadas sus suscripciones y pagos por unidad, debería ser más amplia que la del art. 117, por ejemplo, pasando del $5 \%$ al $10 \%$ de los ingresos, tal y como apunta el Consell de l'Audiovisual de Catalunya CAC en adelante) (2020: 21), pero incluyendo series y otras, habida cuenta las preferencias del mercado.

\section{b) Continuidades negativas acentuadas}

No solo no revaloriza el lugar del servicio público en el nuevo contexto, sino que escora definitivamente el desequilibrio, ya muy notable entre modelos público y privado, en perjuicio del pluralismo y en beneficio de estos últimos en todas las formas imaginables (abierto, pago, OTT, intercambio de videos...). A tenor de sus múltiples sugerencias la CNMC (2020: 59) hace una lectura crítica sobre el tratamiento del servicio público en el APL al reclamarle que haya una reserva de entre 20 a $25 \%$ para el espectro al servicio público y a la comunicación comunitaria en cada ámbito de cobertura, así como la inclusión de medidas para garantizar la prominencia de los canales de servicio público estatal y autonómicos en las plataformas de pago que hagan uso del derecho de must offer.

El Gobierno PSOE-UP no corrige el régimen de "gestión indirecta" en TV autonómica (Murcia, Baleares, Aragón), o sea, de privatización del servicio público en las áreas principales, que incluyó el Gobierno Rajoy, sino que lo ampara y lo extiende también a la TDT local. Es decir, apuesta por la privatización de la comunicación de proximidad $\mathrm{y}$, al mismo tiempo, la ignora como espacio.

La radio y televisión comunitarias (arts. 48,80 y disposición transitoria tercera) son reguladas de forma restrictiva y discriminatoria con reglas distintas (no cabe vender ni alquilar), sigue dependiendo de planes técnicos previos y, además, sin publicidad. De un lado, lo razonable es que siendo competencia totalmente autonómica 
deje hacer a las autonomías pero facilitándoles el uso de espectro. De otro lado, para que subsistan deberían poder acceder a patrocinios y a publicidad institucional, de economía social, del tercer sector y, con ciertos límites, a la local.

c) Ausencias graves

En el APL no se desarrollan los derechos ciudadanos ni siquiera el constitucional (art. 20) "derecho a la comunicación" -era la ocasión- salvo dentro de las obligaciones de los prestadores, cuando aquellos deberían tener preeminencia sobre estas. Con ello hay un desequilibrio entre los principios y los derechos que -como no garantizados- se subordinan a la interpretación y cumplimiento que decidan los prestadores, y más cuando los mecanismos de control planteados tienen fallas. Tampoco se regula el constitucional (20.3) derecho de acceso de la sociedad civil y grupos sociales a los medios públicos.

También son difusos los derechos de los usuarios sin que puedan hacer valer los principios de diversidad y de pluralismo interno más que ante el servicio público. Se deja para códigos de conducta y autorregulaciones, siendo una ocasión perdida para concretar los señalados por ejemplo en la Ley se Protección de Datos (2018).

La propia CNMC considera ausente un cierto desarrollo del pluralismo interno entendido como tratamiento legal para promover "Una amplia gama de valores, opiniones, información e intereses sociales, políticos y culturales que encuentran expresión en los medios de comunicación" (Consejo de Europa). Por ejemplo, el APL no obliga a disponer de Consejos de Profesionales, tan comunes en Europa y en algunos medios.

Se dejan demasiados temas (igualdad de géneros..) a la auto-regulación en lugar de ser directamente regulados y exigibles, con lo que se pueden aplazar sine die en muchos medios de comunicación. Por ello hay un escaso desarrollo de la igualdad de género.

Lo lógico para animar la competencia en el mercado hubiera sido reducir a 10 años el período de duración de las licencias de RTV fijado en 15 años, y eliminar el sistema de renovación automática e indefinida de las mismas en los casos de RTV lineal. Sería razonable que las licencias pasaran luego al mercado o a nuevos concursos.

\section{d) Ausencias poco explicables}

Uno de los motivos de la ley -intercambio de vídeos- no se regula suficientemente. Es chocante. De hecho, menciona pero no regula derechos y obligaciones para los Contenidos Generados por los Usuarios, cuando los servicios a través de plataformas deberían considerarse servicios de comunicación audiovisual, sujetos a las mismas obligaciones. Los Usuarios Generadores de Contenidos, youtubers e infuencers deberían quedar dentro de la norma y cumplir con los principios generales de la comunicación audiovisual y las obligaciones específicas de protección del menor y del consumidor (transparencia, separación e identificación en los servicios entre publicidad y otros). Lo mismo cabe decir de las plataformas de intercambio de vídeos como tal, independientemente de qué distribuyen, cuando habría que evitar que queden fuera de regulación, como dice Alejandro Perales (AAVV, 2021).

Tampoco se regulan los intercambios de audios a pesar de que Spotify compite con radio-fórmulas; ni se desarrolla la normativa sobre agregación de servicios de comunicación audiovisual (registro, límites de dignidad humana, género, menores...) 
La CNMC señala que la cuota de obra europea debería computarse no para el conjunto de los servicios de un prestador, sino para el contenido de cada uno de sus canales o de cada uno de sus catálogos (pg 43).

No desarrolla el concepto de prominencia destacado por la Directiva. Apenas si aparece salvo para la visibilidad en plataformas a petición en relación al cumplimiento de las ofertas obligatorias o de obras europeas. Por ejemplo, la ubicación en prime y day time en abierto, lugar de programas especiales o en lenguas minoritarias, la autopromoción y la posición en catálogo en los canales a petición.

No se señala en el Título IV si las licencias de radio deben renovarse o son automáticas, a diferencia de lo que ocurre con la televisión. Un olvido. Tampoco se señalan límites publicitarios para radio a diferencia de la televisión.

En la televisión de acceso condicionado el 30\% de los contenidos audiovisuales han de ser subtitulados. Sería razonable que en accesibilidad para personas discapacitadas (subitulados, signos, audiodescripción) no tuvieran obligaciones rebajadas las de acceso condicionado y a petición (art. 101) respecto a las convencionales ( $75 \%$ de la programación y $100 \%$ de los emitidos en máxima audiencia, 5 horas semanales en lenguaje de signos y audiodescritos) (art. 100).

En el caso de las plataformas de intercambio de video hay una difícil protección, por no concretada, en el caso de los prestadores que lo hagan desde fuera de la UE.

Respecto a la alfabetización mediática (art. 10) no se ve bien el mecanismo para hacerlo valer, aunque se haría un informe al respecto cada trienio.

El Registro competente de prestadores de servicios audiovisuales debería ser solo el autonómico "cuando éstos tengan su domicilio en una comunidad autónoma y dirijan específicamente sus contenidos a la ciudadanía de aquel territorio" (CAC, 2020: 9)

En el ámbito publicitario hay unas cuantas lagunas. La publicidad institucional no tiene obligaciones de trasparencia respecto a los medios (sí hacia las centrales de compras). No se hace eco apenas de las nuevas formas publicitarias con lo que se puede soslayar los requisitos de un APL, más centrado para los formatos publicitarios convencionales. La propia CNMC (pgs 33 y ss) reclama que la normativa sobre comunicaciones comerciales también se aplique en plataformas de intercambio de vídeos. En otro caso Youtube y otras (Instagram TV, TikTok y Twitch) pueden quedar fuera. Los usuarios deberían poder dar su consentimiento previo a la recepción de publicidad híbrida o interactiva, a través de servicios de televisión conectada, así como desistir de dicho consentimiento mediante un procedimiento simple y gratuito, según la CNMC.

Hay lagunas -el CAC dixit- en el régimen sancionador sobre el incumplimiento de códigos de conducta, o en la obligación de informar de una inclusión publicitaria en las plataformas de intercambio de videos, o sobre los abusos en teletienda, o cuando se sobrepasen parámetros técnicos de emisión, o en el aseguramiento de los contenidos esenciales de la licencia...

Parecería conveniente una revisión del APL en relación a los efectos (horas de visionado, géneros audiovisuales, visionados multisoportes, teletrabajo, restricciones presenciales) y cautelas (publicidad responsable...) que las restricciones sociales y los confinamientos derivados de la Covid han dejado en los usos comunicativos.

\section{e) La diversidad lingüística y cultural en peligro}

La diversidad -lo destaca Trinidad García Leiva (AAVV, 2021)- no es fuente doctrinal en la exposición de motivos ni en los principios básicos, ni tiene desarrollo en el 
articulado - a diferencia del más amplio que tiene el concepto de pluralismo-. Ello es relevante para la visibilidad del audiovisual de los países pequeños europeos y de las comunidades con lengua propia más allá de un gaseoso "promoverá el conocimiento y difusión de la lengua oficial del Estado y las lenguas oficiales de las CCAA y sus expresiones culturales" del art. 8.

Se cita el respeto a la diversidad cultural y comunicativa pero se la confunde con el pluralismo (art. 5) y no se regula. Se reconocen las lenguas minoritarias (art. 8) pero sin compromiso y sin mención a la diversidad (con su carga doctrinal). Hay tanto un problema conceptual como de desarrollo.

La propia CNMC reclama que "Dentro del pluralismo, se propone incluir el derecho a que la comunicación audiovisual incluya una programación en abierto que refleje la diversidad cultural y lingüística de la ciudadanía" (pg. 81) y que "resultaría adecuado que se incluyeran elementos que promovieran la inversión en obras destinadas a promover la diversidad cultural y lingüística, así como en contenidos que además de respetar, contribuyan a los principios generales de la comunicación audiovisual" (pg. 85). Lo cierto es que la diversidad solo se verbaliza pero con poca concreción en obligaciones culturales o lingüísticas ya que no contempla porcentajes precisos para lenguas distintas al castellano ni se plantea la aplicación de la idea de prominencia ni deja espacio para que las CCAA intervengan sobre las plataformas.

Para que, de verdad, se promoviera la diversidad cultural y lingüística, debería reservarse una cuota del $40 \%$ de la inversión obligatoria en obras audiovisuales de las televisiones lineales tanto de servicio público estatal como privadas, a petición y plataformas, a producciones, Fondo de Protección de la Cinematografía y el Audiovisual y derechos de explotación en lenguas minoritarias, habida cuenta que los territorios con lenguas minoritarias cooficiales concentraban en 2020 el 41,29\% de la población española (INE, 2021). El CAC (pg-19) propone el 50\%. Al no señalarse una subcuota, toda ella -con alguna excepción- va e iría por razones de normas discriminatorias y de diglosia a obras en castellano tanto en televisión líneal y a petición como en intercambio de videos, dejando la mención a lenguas cooficiales en la coletilla retórica políticamente correcta, propia del papel mojado.

\section{f) Pasos atrás}

Aparece una visión más liberal y de mínimos en las obligaciones publicitarias en horarios y franjas - con base en la Directiva pero era mejorable- que pasan de $20 \%$ por hora, a 144 minutos de 6:00 a 18:00 y a 72 minutos de 18:00 a24:00, con lo que desaparece la regulación por hora y por franja, con riesgos de saturación en prime time y day time, lo que genera, a medio plazo y por aburrimiento, una clientela potencial para las plataformas. Sin límite, se añaden marcos neutrales, autopromociones, patrocinio, emplazamientos de productos, promoción de la cultura europea y anuncios de servicio público.

El APL no se centra en asegurar al máximo la pertinencia de contenidos, calidad, origen de la oferta, su naturaleza, número de actores o nuevos valores, como los bienes comunes. Se limita a establecer unos mínimos legales y unas reglas de tráfico entre operadores.

En orden a la protección de menores y a ciertas obligaciones en las plataformas de intercambio, el APL es demasiado genérico respecto a algunos contenidos (pornografía, violencia, dignidad). Aunque se deba separar contenido y publicidad y exige 
que se cree un sistema de calificación de contenidos, no se ve claro el mecanismo de vigilancia que se habilitaría para reconducirlos en su caso.

De hecho, aunque extiende las obligaciones a los servicios a petición protege menos en la televisión convencional a los y las menores que la ley de 2010, al eliminar los horarios de protección reforzada - se sustituyen por "primarán" programas infantiles en horarios de 7:30 a 9:00 en laborables y 7:30 a 12:00 en sábados y festivos dice el art. 97- pudiéndose, además, interrumpir los programas infantiles, transcurrida media hora, con publicidad, según el art. 136.3 (ver postdata). Sí se incluye como necesario el etiquetado de contenidos (pero sin precisiones) y se invita a mecanismos de identificación y de control parental mientras prohíbe la generación de perfiles comerciales. Sería conveniente homogeneizar y simplificar en un mismo sistema de calificación tanto para el servicio lineal como para audiovisual a petición y plataformas, especialmente para estas dos últimas al visionarse sin horarios. $\mathrm{Al}$ mismo tiempo para el servicio lineal -que sigue estando presente incluso a través de las plataformas- deberían mantenerse los horarios de exclusión entre las 6:00 a 22:00, tal y como reclama la Asociación de Usuarios de la Comunicación (2021).

Hay alguna invasión de competencias en distintas cuestiones según señala el CAC: definición de los órganos de gobernanza y gestión del servicio público de comunicación audiovisual (art. 55) que son competencia autonómica; deja fuera a las comunidades de la supervisión de los intercambios de video...

Igualmente, tal y como alega el CAC (año), "Sería conveniente que en las funciones atribuidas a la CNMC en los artículos 92 (supervisión y control de las plataformas de las plataformas de intercambio de vídeos), 96 (calificaciones de los programas), 118 (control de cuotas de obras europeas), 148 (plan estratégico audiovisual), 149 (fomento del sector audiovisual) y 153 (competencias de supervisión, control y sancionadoras), así como en la disposición final primera, en cuanto a los puntos 2 , $8,9,10,15$ y 16 , se previera que las funciones atribuidas a la CNMC se ejercerán en régimen de colaboración con las autoridades independientes de regulación de las comunidades autónomas" (pg.3). Así como su participación en el europeo ERGA (European Regulators Group for Audiovisual Media Services).

Lo que, en definitiva, es un llamamiento para que cada comunidad disponga de un Consejo independiente del Audiovisual, lo que solo ocurre en Catalunya y Andalucía.

Se renuncia a crear el CEMA y se le asigna a la CNMC una función ampliada (control del pluralismo, de contenidos audiovisuales, de las faltas graves o muy graves..) que no puede ejercer. Llama la atención que de las ocho funciones que se le asignan solo una se refiera al pluralismo y están ausentes temáticas como los bienes comunes, producción propia, descentralización, calidad de la información, seguimiento del servicio público... Además del desbordamiento funcional ha tenido una incapacidad histórica, por falta de recursos, para la tramitación y ejecución de sanciones (entre 2013-2017 solo recuperó el 3\% según Europa Press, 2017). Estaba mejor regulado el CEMA en la ley de 2010.

El Ministerio de Asuntos Económicos y Transformación Digital, se reserva el rol de auténtico regulador, a contrapelo de la UE. Es la "Autoridad Audiovisual competente". El Consejo de Ministros se reserva la potestad para aprobar las bases de la convocatoria del concurso para el otorgamiento de licencias y la competencia para otorgar los títulos habilitantes así como otras funciones. No obstante, un voto particular de dos consejeros de la CNMC (pg 93) se hace eco de que una Recomendación 
(23, del 2000) del Consejo de Europa señala que sea la Autoridad Audiovisual independiente quien otorgue las licencias.

\section{Diagnóstico general}

Como valoración general, es una oportunidad perdida ya que se enfoca como mera trasposición lineal y conservadora de la Directiva. En ningún tema supera los mínimos de la Directiva, que autoriza siempre a ir más allá en las legislaciones estatales, pero este APL tiene la vocación de no tocar en ningún momento los intereses de los operadores clásicos y de molestar poco a las plataformas con obligaciones superiores a las del legislador europeo.

Supone una continuidad y profundización del modelo mercantilista, privatista y parcialmente desregulado de la Ley de 2010, como expresión, también, del retroceso doctrinal europeo sobre la función social de las comunicaciones, en contraste con las teorías de los bienes comunes y de la función innovadora del Servicio Público. El texto concibe casi solo el ámbito audiovisual como negocio y la RTV y la comunicación como mercancías de las que solo hay que regular el tráfico competitivo entre los pocos actores (oligopolios) que quedan, eso sí con algunas obligaciones, siempre descendentes. Encaja en la tradición privatizadora y de aceptación del deterioro del lugar social del servicio público y que, si existe, es menos por comprensión de su rol, que por cierta tradición europea que no ha existido como tal en España.

Hay rebajas de obligaciones en el campo de la comunicación comercial para todos. Para no exigirles a los nuevos partenaires, se les reducen las restricciones a todos los prestadores, como dice Alejandro Perales (AAVV, 2021).

La visión de la comunicación audiovisual y sus servicios sigue siendo (ver la exposición de motivos) la de "servicios en competencia" y solo adicionalmente "herramienta de transmisión de valores" y con virtualidad "para el ejercicio de la libertad de expresión". El APL se propone actualizar el marco jurídico para seguir la evolución del mercado, con el angelical propósito de buscar el "equilibrio entre acceso, producción del usuario y competencia".

Se parte -sin tocarlos- de los triopolios de televisión en abierto y de plataforma (Atresmedia, Mediaset, $\mathrm{M}+$ ) y de radio (Ser, Cope y Once acaparan el 70\% de la audiencia) y de las plataformas tecnológicas existentes. La falta de pluralismo no es entendida como problema. Ni garantiza que tampoco vaya a más, como ya ocurre en las redes protocolizadas.

Hay una decepcionante regulación de las plataformas de intercambio de vídeos que, al igual que el streaming, tampoco están sometidas a licencia pues no se ven afectadas por la escasez del espectro. Se limita a temas de menores, dignidad y temas publicitarios, sin obligaciones de promoción audiovisual ni de financiación. Se trata de una discriminación respecto a los operadores convencionales. Siendo imposible fijar cuota europea, no se entiende que no se les exija contribuir a la financiación anticipada de obra europea, dado que se aprovechan del saber audiovisual y del ecosistema audiovisual (ver postdata).

Por tanto no se trata de un APL garantista de derechos de los usuarios -no están en el centro de la normativa- ni es promotor de nuevos operadores y nuevos contenidos que multipliquen los prestadores de servicios en aras al pluralismo, sino simplemente de señalamiento de unas pocas obligaciones para los operadores vigentes. 
Es un misterio que el gobierno más de izquierda de la historia de los últimos 80 años, y con mayoría parlamentaria asequible, proponga semejante ley. ¿Cabe teorizar sobre la debilidad, cuando no la inconsistencia e ineptitud, de la democracia ante el poder de la plutocracia de plataformas y operadores que tendrían cautivas para sus intereses a las Administraciones públicas?

Es manifiestamente mejorable pero no orientable en otra dirección desde sus mimbres de partida.

POSTDATA: Hay cambios de última hora ministeriales mediante modificaciones puntuales del anteproyecto para las segundas alegaciones que acaban el 12 de julio y que corrigen algunas cuestiones aquí planteadas. Entre otros cambios se incluye una nueva Disposición Final Primera de modificación general de la Ley 8/2009 de financiación de RTVE. Entre otras cuestiones Netflix, Disney, Youtube... dedicarían 1,5\% de sus ingresos en España a la financiación de RTVE y las operadoras de telecomunicaciones, en lugar de una aportación directa del $0,9 \%$ de sus ingresos al que venían obligados para ese menester, lo harían mediante la tasa por uso del espectro radioeléctrico, lo que convendría cotejar para saber cómo le deja a RTVE el cambio. Una nueva Disposición Adicional Tercera prevé el fomento de la producción de obras audiovisuales dirigidas o producidas por mujeres en las convocatorias de ayudas con cargo al Fondo de Protección a la Cinematografía y al Audiovisual. Según Vertele con fuentes de Europa Press (30-6-21) se modificarán los porcentajes de inversión para la promoción de obra europea y se prevé que para noviembre de 2021 pueda entrar como proyecto en el Congreso de los Diputados

\section{Referencias}

AAVV (2021) "La futura Ley de Comunicación a debate" (11-3-2021). Webinario de la AEIC-, en el que participaron Alfonso Albornoz, Manuel Chaparro, Ángel García Castillejo, Trinidad García Leiva, Ana Azurmendi, Carmina Crusafon, Javier García, Alejandro Perales.... Ver http://diversidadaudiovisual.org

Anteproyecto de Ley General de Comunicación Audiovisual (Ministerio de Asuntos económicos y Transformación Digital- Secretaria de Estado de Telecomunicaciones e Infraestructuras Digitales) de 2020. Ver https://avancedigital.mineco.gob.es/es-es/Part. icipacion/Paginas/DetallePart.icipacionPublica.aspx?k=355

Anteproyecto de Ley General de Comunicación Audiovisual de 2020, Nueva versión a junio 2021. https://portal.mineco.gob.es/RecursosArticulo/mineco/ministerio/participacion_ publica/audiencia/ficheros/210628-APL-Com-Audiovisual-2.pdf

Asociación de Usuarios de la Comunicación. Alegaciones al anteproyecto. Ver https://www. auc.es

Bustamante Enrique (2020) "Un Gobierno progresista, pero neoliberal en lo audiovisual". El diario.es 25-12-2020

Comisión Nacional de los Mercados y la Competencia. Alegaciones al anteproyecto. Ver https://www.cnmc.es/expedientes/ipncnmc04220

Council of Europe- Committee of Ministers (2000) "Recommendation Rec(2000)23 of the Committee of Ministers to member states on the independence and functions of regulatory authorities for the broadcasting sector". Ver https://search.coe.int/cm/Pages/ result_details.aspx?ObjectId $=09000016804 \mathrm{e} 0322$ 
Ley General de Comunicación Audiovisual (2010) https://www.boe.es/buscar/act. php?id=BOE-A-2010-5292

Plataforma de Defensa de la Comunicación y el Periodismo de Andalucía. Alegaciones al anteproyecto. Ver https://www.emart.v.es/r1_pdfs/PDCPA-ALEGACIONES-APL.pdf

Vertele "La nueva Ley Audiovisual hará que RTVE sea financiada por las plataformas igual que por las cadenas privadas". Ver https://vertele.eldiario.es/noticias/nueva-leyaudiovisual-hara-rtve-sea-financiada-plataformas-igual-cadenas-privadas_1_8089820. html

Zallo Ramón (2011) "Los desarrollos normativos en comunicación audiovisual 20042010. El giro neoliberal del gobierno socialista", en Estructuras de la comunicación y la cultura. Políticas para la era digital. Barcelona: Gedisa. 